\title{
INCIDENCE OF SYMPTOMATIC INTERNAL HERNIA FOLLOWING MINIMAL INVASIVE GOLORECTAL SURGERY: A SINGLE CENTER EXPERIENGE
}

\author{
Rajiv Nakarmi, ${ }^{1}$ Tian Yu-Feng, ${ }^{2}$ Khaa-Hoo Ong, ${ }^{2}$ Muza Shrestha, ${ }^{1}$ Sundar Maharjan, ${ }^{1}$ \\ Suman Bikram Adhikari Chhetri ${ }^{1}$
}

${ }^{1}$ Department of General and GI Laparoscopic Surgery, Manmohan Memorial Medical College and Teaching Hospital, Swoyambhu, Kathmandu, Nepal and ${ }^{2}$ Chi Mei Medical Center, Tainan City, Taiwan

\begin{abstract}
Laparoscopy has been adopted in the surgical specialties and colorectal surgery for treatment of benign and malignant diseases. Recent reviews suggest that the incidence of symptomatic internal hernias after laparoscopic colorectal resection is from 0.39 to $0.65 \%$. Unlike in open surgery, laparoscopic closure of a mesenteric defect is inherently challenging as inadvertent injury to the marginal vessels may compromise blood supply to the anastomosis. For these reasons, many surgeons leave the defect open during laparoscopic surgery. But this may lead to development of post-operative internal hernia through the defect. This is a retrospective study where we included 149 patients who underwent laparoscopic/ robotic colorectal surgeries from March 2019 to March 2020. Data pertaining for following variables were collected which included age, sex, indication for surgery, location of the pathology, splenic flexure mobilization. The incidence of internal hernia among these patients were calculated and assessed using SPSS 20 . Incidence of internal hernia was found to be $0.67 \%$ which was diagnosed and treated on the $18^{\text {th }}$ post-operative day of initial surgery. Internal hernia is a rare but important complication of laparoscopic/robotic colorectal surgery with a high mortality rate if not diagnosed early. Defect closure is still controversial during the initial surgery and probably not indicated for all patients and depends on surgeon's preference.
\end{abstract}

\section{KEYWORDS}

Minimal invasive colorectal surgery, internal hernia, laparoscopy

Received on: March 05, 2021

Accepted for publication: July 18, 2021

\section{CORRESPONDING AUTHOR}

Dr. Rajiv Nakarmi,

Junior Consultant,

Department of General and GI Laparoscopic Surgery, Manmohan Memorial Medical College and Teaching Hospital, Swoyambhu, Kathmandu, Nepal

Email: rajivnakarmi31@gmail.com

Orcid No: https://orcid.org/0000-0003-2205-1784

DOI: https://doi.org/10.3126/nmcj.v23i3.40377 


\section{INTRODUCTION}

Laparoscopic technique is being adopted in various surgical specialties including colorectal surgery for treatment of benign and malignant disease. ${ }^{1}$ Laparoscopic colorectal surgery has gained popularity since first described by Jacobs. ${ }^{2}$ Several studies have documented better short-term outcomes of laparoscopic colectomy as compared to conventional open colectomy. ${ }^{3-6}$ Additional benefits of laparoscopic colorectal resection including less pain, faster recovery of bowel function and decreased length of stay, have contributed to this approach becoming widely used. ${ }^{7}$ Specific complications unique to laparoscopic surgery have also been reported, including port-site hernias, ${ }^{8}$ and leg compartment syndrome after prolonged Trendelenburg position in the lithotomy position, ${ }^{9}$ Several reports have shown that laparoscopic colectomy is associated with a lower incidence of small bowel obstruction than open colectomy. The reported rates of postoperative small bowel obstruction after laparoscopic colectomy and open colectomy are $2.0-7.8 \%$ and $3.0-18.3 \%$, respectively. ${ }^{1,10,11}$

Postoperative intestinal obstruction is mainly caused by adhesions of the small intestine, but may rarely be caused by an internal hernia projecting through a post-colectomy mesenteric defect. ${ }^{12}$ Recent reviews suggest that the incidence of symptomatic internal hernias after laparoscopic colorectal resection is from 0.39 to $0.65 \%,{ }^{12,13}$ and $64.3 \%$ of the cases occurred after left sided resection. ${ }^{12}$ Unlike in open surgery, laparoscopic closure of a mesenteric defect is inherently challenging and inadvertent injury to the marginal vessels may compromise blood supply to the anastomosis. ${ }^{14,15}$ For these reasons, many surgeons leave the defect open during laparoscopic surgery. ${ }^{16}$ Incomplete closure of the mesenteric defect may leave a narrow residual defect, which may actually increase the risk of internal hernia. ${ }^{17}$ Ichimura et $a .^{18}$ reported a case of internal hernia through the mesenteric opening rimmed with the mesocolon and preserved superior rectal artery (SRA) after laparoscopic left colectomy.

\section{METHODS AND METHODOLOGY}

Patient selection: This is a retrospective study including 149 patients who underwent laparoscopic / Da Vinci colorectal surgeries including, right hemicolectomy, left hemicolectomy, laparoscopic high anterior/ lower anterior resection, laparoscopic assisted TaTME (Trans-anal Total Mesorectal Excision) at Chi Mei Medical Centre, Tainan from
March 2019 to March 2020. Data pertaining for following variables were collected which included age, sex, indication for surgery, location of the pathology. The study was approved by Institutional Review Board of Chi Mei Medical Center, Tainan City, Taiwan.

Surgical Technique: Adequate preoperative workup was done followed by preoperative bowel preparation was done. In few cases for easy localization of the tumor site colonoscopy and tattooing was done.

Patient was positioned in Lloyd-Davis position. In case of right hemicolectomy, the surgeons operated from the left side where as in left hemicolectomy, sigmoidectomy/ lower anterior resection the surgeon operated from the right of the patient. Initial laparoscopy evaluated the carcinomatosis, adjacent organ involvement and liver condition.

Laparoscopic colectomy was performed using a medial-to lateral approach in all patients, no touch technique and early and proximal ligation of lympho-vascular pedicle. The vessels ligated during right hemicolectomy was ileocolic, right colic, right branch of middle colic after the identification of duodenum, ureter. Left hemicolectomy, sigmoidectomy, high anterior resection and lower anterior resection were performed by high ligation of inferior mesenteric artery and vein, with lymph node dissection. However, for benign conditions root of inferior mesenteric artery was not divided. Splenic flexure mobilization was carried out in anterior and lower anterior resection and few cases of sigmoidectomy according to the tension in the line of anastomosis. The dissection for each level of colectomy was carried out by standard protocol. Right and left hemicolectomies were performed with extracorporeal anastomosis.

Statistical Analysis: The statistical analysis was carried out by using SPSS 20 .

\section{RESULTS}

Age distribution ranged from 40 years to 92 years with mean age of $65.46+/-11.4$ years (Fig. $1)$. Among the cases $41(26.3 \%)$ of them were of female gender and 108(73.3\%) were male. The location of the tumor showed that most of the tumors were located at the sigmoid colon accounting for $47(32.2 \%)$ of cases and rectum accounting for $42(28.2 \%)$ of cases (Fig. 2).

Fig. 3. Illustrates the types of surgeries that were performed. Majority of surgery was laparoscopic anterior resection which 


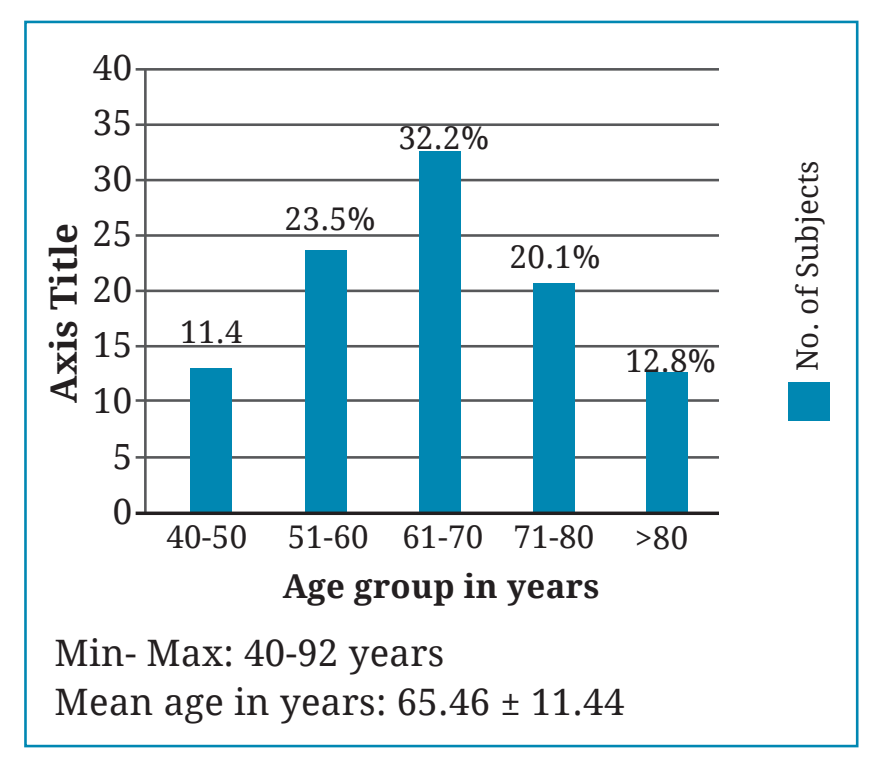

Fig. 1: Age distribution of subjects $(\mathrm{N}=149)$

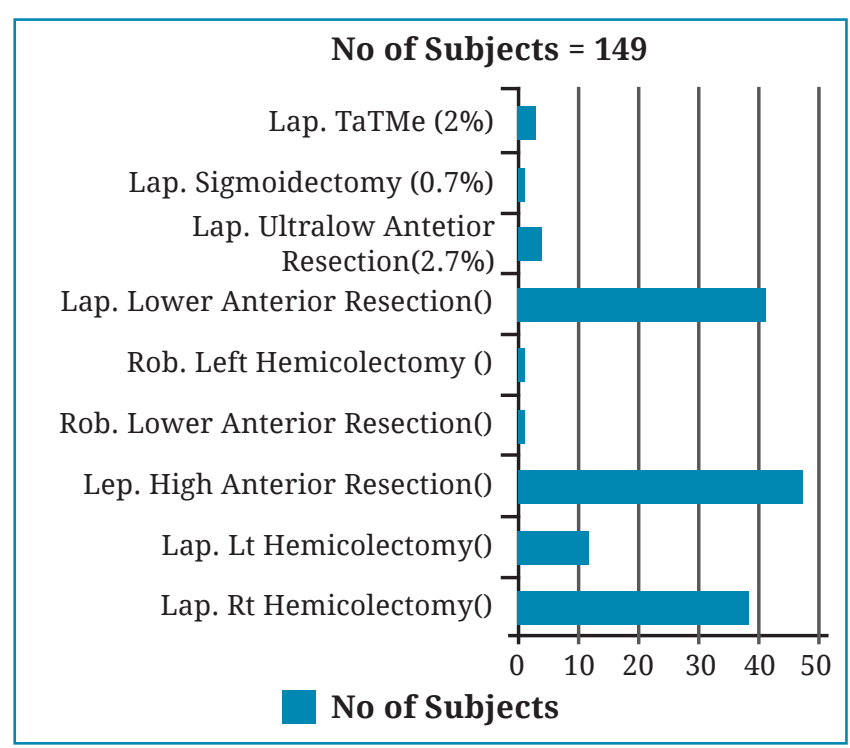

Fig. 3: Distribution of procedures

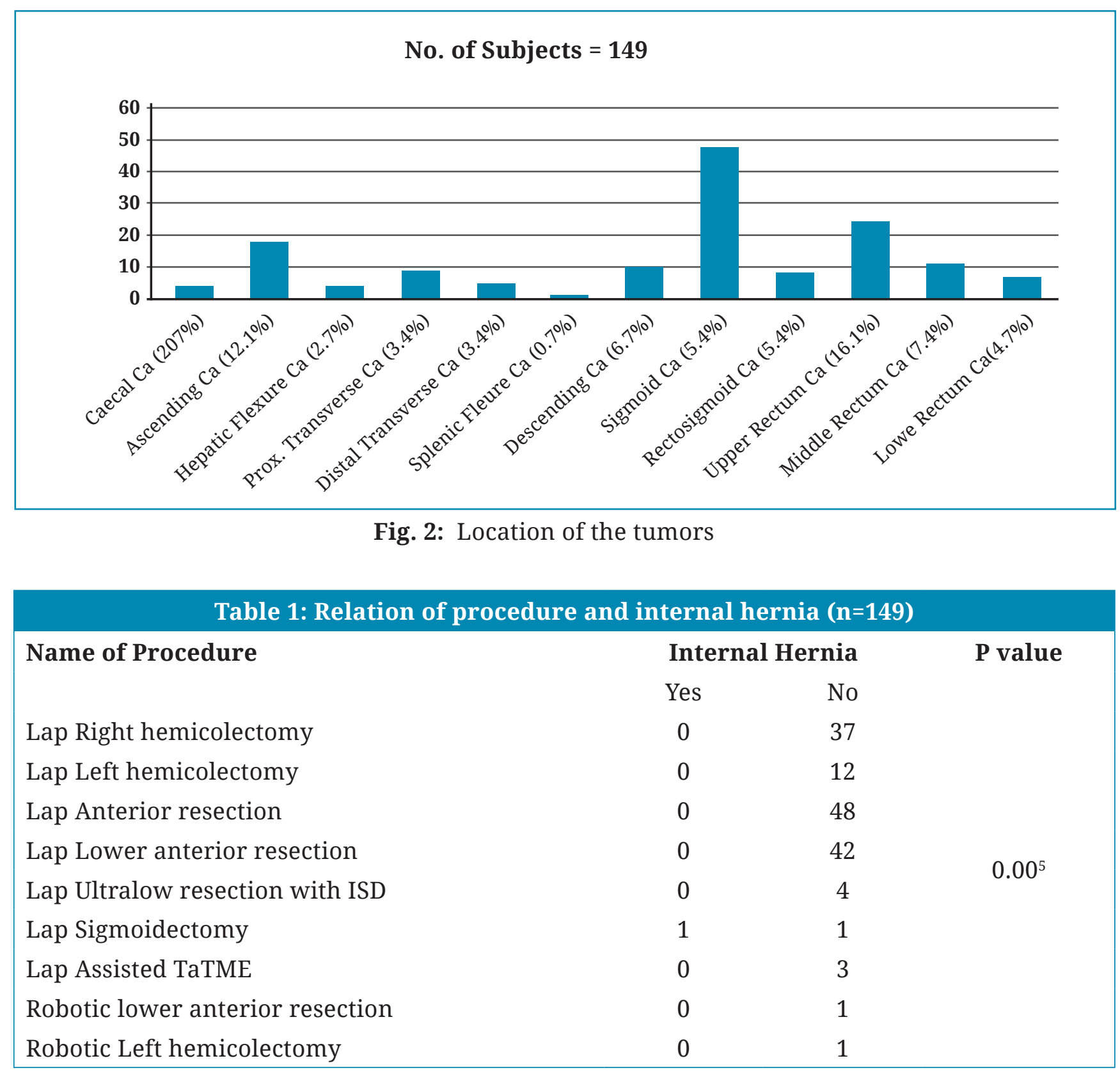


accounted for 99(66.5\%) of cases (high, low, ultralow and TaTME). Among the patients, 1 patient $(0.67 \%)$ developed post-operative internal herniation with $\mathrm{p}$ value of 0.00 which is significant $(<0.05)$ as shown in Table1.

\section{DISCUSSION}

Laparoscopic surgery now has proved that it's as more effective as the open surgery and it's used for the management of colon cancer

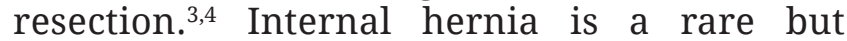
fatal complication after laparoscopic colonic resection. To confirm this diagnosis, it requires emergency re-operation because most of the patients come with non- specific symptoms.

Among the 149 cases of laparoscopic colonic surgeries in this study, it was found that 1 patient $(0.67 \%)$ developed intestinal obstruction features due to internal herniation. He underwent surgery for sigmoid diverticulosis where laparoscopic sigmoidectomy was carried out with high ligation of IMA as the bowel wall and mesentery was edematous and the involved segment was large. The splenic flexure was mobilized adequately to facilitate tension free anastomosis between the descending colon and upper rectum. His postoperative period was uneventful and was discharged. On $18^{\text {th }}$ postoperative day he presented in emergency department with complaints of poorly localized intermittent pain abdomen which increased in severity for 1 day. On examination he had generalized distension of abdomen with mild tenderness but no features of peritonitis. Plain Computed tomography of abdomen showed features suggestive of small bowel obstruction. Laparoscopic exploration revealed there was an internal herniation of small bowel through the defect of mesocolon and the small bowel ascended up to the left peri-splenic area posterior to the descending colon.

In a meta-analysis done in 2019 by Giuseppe Portale et al, occurrence rate of internal hernia after laparoscopic colorectal resection is around 5 per 1000 patients $(0.5 \%) .{ }^{13}$ Similarly other reviews suggest that the incidence of symptomatic internal hernias after laparoscopic colorectal resection is from 0.39 to $0.65 \%,{ }^{12,13}$ and $64.3 \%$ the cases of internal hernia occurred after left sided resection. It also states that small-sized studies are likely to overestimate the occurrence of internal hernia. This study included 149 cases where the incidence was $0.67 \%$. Most reported cases of internal herniation occurred early in the post- operative period, ${ }^{19}$ but may occur even after months to years after initial surgery. ${ }^{20}$ In this study it occurred at around 18 days after the initial operation.

Several mechanisms have been proposed to explain the development of internal hernia after laparoscopic colorectal resection. Minimally invasive surgery minimizes postoperative adhesions, thus reducing the chance for mechanical obstructions from adhesive bands, but also increases the risk of SBO by promoting free movement of ileal loops in the abdominal cavity - even below the mesentery - if the defect created with colonic resection is not closed. ${ }^{10}$ Further, laparoscopy, with reduced postoperative pain, compared to open procedures, especially with recent widespread difusion of Enhanced Recovery After Surgery (ERAS) principles, promotes early mobilization. This prevents the adherence of the neo-descending colon to the Gerota's fascia. ${ }^{32}$ Several mechanisms have been proposed to explain the development of internal hernia after laparoscopic colorectal resection. Minimally invasive surgery minimizes postoperative adhesions, thus reducing the chance for mechanical obstructions from adhesive bands, but also increases the risk of SBO by promoting free movement of ileal loops in the abdominal cavity - even below the mesentery - if the defect created with colonic resection is not closed. ${ }^{10}$ Further, laparoscopy, with reduced postoperative pain, compared to open procedures, especially with recent widespread dilusion of ERAS principles, promotes early mobilization. This prevents the adherence of the neo-descending colon to the Gerota's fascia. ${ }^{32}$ Several mechanisms have been proposed to explain the development of internal hernia after laparoscopic colorectal resection. Diagnosing internal hernia can be difficult due to varying symptoms ranging from significant discomfort or constant vague pain to intermittent diffuse abdominal pain. Same clinical manifestation was seen in the case. In suspected cases, internal hernia can also be demonstrated radiographically on either conventional X-rays, or more frequently, on computed tomography (CT). On CT, internal hernia is characterized by mesenteric vessel abnormalities such as vessel crowding, twisting, and stretching. The bowel loops may be distended or located in the hernia sac/ mesenteric defect. ${ }^{21}$ Abdominal imaging including GI series with barium enema may also be helpful. ${ }^{21}$ Although several typical CT findings have been described including "U" or "C" shaped small bowel loops postero-lateral to the left neo-descending colon after left sided colorectal surgery, their absence doesn't warrant absence of internal hernia. ${ }^{19}$ In this case 
CT scan showed only dilated bowel loops with transition point at peri-umbilical region. Bowel ischemia is the most severe presentation of $\mathrm{IH}$ with a mortality of $20-50 \% .{ }^{21}$ Fewer adhesions after LCRS (Laparoscopic Colorectal Surgery) potentially increase the risk of internal hernia through the mesenteric defect. Repositioning and tilting of patients during LCRS and early postoperative mobilization may also increase that risk. Mobilization of the ligament of Treitz and splenic flexure have been described as risk factors for left-sided resections. Small bowel mesentery which is anchored to the posterior abdominal wall from posterior to anterior and from left side to right, has a natural tendency to lie in left iliac fossa and hence slide below and left of mesocolon. ${ }^{19}$ Mobilization of splenic flexure can contribute to prevention of internal hernia. If it's mobilized incompletely then there is a tension in the mesocolon thus preventing ileum to slide under the defect but if it does slide then the complication may be life threatening. ${ }^{13}$ However, mesenteric defects were not routinely closed during initial LCRS. Although routine closure of mesenteric defects has been suggested by some authors, closure attempts during LCRS can be technically difficult and time-consuming. In addition, incomplete closure may leave a narrow residual defect that could paradoxically increase SBO risk, and it should be kept in mind that closure could compromise perfusion of anastomoses. ${ }^{20}$

Currently there is no any strong evidence which suggest routine closure of the defect and it depends on the operating surgeon's hand and laparoscopic skills. Several techniques have been described to close the defects such as running sutures with non-absorbable suture, clips, fibrin glue being the most common. ${ }^{12,22}$ However there are various issues regarding closure of the defect. It is time consuming, technically challenging, increased risk of damaging the retroperitoneal structures, or damaging the marginal arteries which may lead to anastomosis leak. ${ }^{23}$ So some surgeons suggest simple positioning the small bowel to right of mesocolon for left colorectal resection and vice-versa with or without omental interposition media to the mobilized colon. ${ }^{23}$

This study has small number of patients and that is the reason for our result of patient developing internal hernia after laparoscopic colorectal surgery is slight more than $0.5 \%$ that is $0.67 \%$.

Internal hernia is a rare but important complication of laparoscopic colorectal surgery with a high mortality rate if not diagnosed early. Internal hernia should be suspected when patients do not recover as expected. More efforts should be made to identify risk factors of internal hernia as this could indicate which patients would benefit from closure of mesenteric defects during laparoscopic colorectal surgery. Further comparative study with adequate data is required.

Conflict of interest: None

Source of research fund: None

\section{REFERENCES}

1. Davis CH, Shirkey BA, Moore LW et al. Trends in laparoscopic colorectal surgery over time from 2005-2014 using the NSQIP database. J Surg Res 2018; 223: 16-21.

2. Jacobs M, Verdeja JC, Goldstein HS. Minimally invasive colon resection (laparoscopic colectomy). Surg Laparosc Endosc 1991; 1: 14450.

3. Nelson H, Sargent DJ, Wieand HS et al. A comparison of laparoscopically assisted and open colectomy for colon cancer: Clinical Outcomes of Surgical Therapy Study Group. $N$ Engl J Med 2004; 350: 2050-9.

4. Lacy AM, García-Valdecasas JC, Delgado S et al, "Laparoscopy-assisted colectomy versus open colectomy for treatment of non-metastatic colon cancer: a randomised trial.," The Lancet 2002; 359: 2224-9.

5. Guillou PJ, Quirke P, Thorpe H et al. Short-term endpoints of conventional versus laparoscopic- assisted surgery in patients with colorectal cancer (MRC CLASICC TRIAL): multicentre, randomised control trial. Lancet 2005; 365: 171826.

6. Colon Cancer Laparoscopic or Open Resection Study Group, Buunen M, Veldkamp R, Hop WC et al. Survival after laparoscopic surgery versus open surgery for colon cancer: long-term outcome of a randomised clinical trial. Lancet Oncol 2009; 10: 44-52.

7. Nelson H, Sargent DJ, Wie HS et al. A comparison of laparoscopically assisted and open colectomy for coloncancer. N Engl J Med 2004; 350: 2050-9.

8. Plaus WJ. Laparoscopic trocar site hernias. $J$ Laparoendosc Surg 1993; 3: 567-70.

9. Soop M, Carlson GL. Well leg compartment syndrome after prolonged pelvicsurgery: launch of United Kingdom and Ireland multidisciplinary clinicalguidelines. Dis Colon Rectum 2019; 62: 1150-2. 
10. Kitano S, Inomata M, Mizusawa J et al. Survival outcomes following laparoscopic versus open D3 dissection for stage II or III colon cancer (JCOG0404): a phase 3, randomised controlled trial. Lancet Gastroenterol Hepatol 2017; 2: 2618.

11. McKay GD, Morgan MJ, Wong SK et al. Improved short-term outcomes of laparoscopic versus open resection for colon and rectal cancer in an area health service: a multicenter study. Dis Colon Rectum 2012; 55: 42-50.

12. Toh JW, Lim R, Keshava A, Rickard MJ. The risk of internal hernia or volvulus after laparoscopic colorectal surgery: a systematic review. Colon Dis 2016; 18: 1133-41.

13. Portale G, Popescu GO, Parotto M, Cavallin F. Internal hernia afterlaparoscopic colorectal surgery: an under-reported potentially severecomplication. A systematic review and meta-analysis. Surg Endosc 2019; 33: 1066-74.

14. Cabot JC, Lee SA, Yoo J et al. Long-term consequences of not closing the mesenteric defect after laparoscopic right colectomy. Dis Colon Rectum 2010; 53: 289-92.

15. Ansari N, KeshavaA, Rickard MJ et al. Laparoscopic repair of internal hernia following laparoscopic anterior resection. Int'l J Colorectal Dis 2013; 28: 1739-41.

16. Lee SY, Kim CH, Kim YJ et al. Internal hernia following laparoscopic colorectal surgery: a rare but fatal complication. Hernia 2016; 21: 299-304.
17. Masubuchi S, Okuda J, Tanaka $\mathrm{K}$ et al. Internal hernia projecting through a mesenteric defect to the lesser omental cleft following laparoscopicassisted partial resection of the transverse colon: report of a case. Surg Today 2013; 43: 814-7.

18. Ichihara $\mathrm{T}$, Morita $\mathrm{T}$, Fujita $\mathrm{F}$ et al. Internal hernia through the mesenteric opening rimmed with the mesocolon and the preserved inferior mesenteric artery after laparoscopic-assisted left colectomy: report of a case. J Jpn Soc Endoscopic Surg 2011; 16: 709-13.

19. Sereno Trabaldo S., Anvari M, Leroy J, Marescaux J. Prevalence of internal hernias after laparoscopic colonic surgery. J Gastrointest Surg 2009; 13: 1107-10.

20. Svraka M, Wilhelmsen M, Bulut O. Internal hernia following laparoscopic colorectal surgery: single center experience. Polski Przeglad Chirurgiczny 2017; 89: 19-22.

21. Martin LC, Merkle EM, Thompson WM, Review of internal hernias: radiographic and clinical findings. Am J Roentgenol 2016; 186: 703-17.

22. Angelini P, Sciuto A, Cuccurullo D, Pirozzi F, Reggio S, Corcion F. Prevention of internal hernias and pelvic adhesions following laparoscopic left sided colorectal resection: the role of fibrin sealant. Surg Endosc 2017; 31: 3048-55.

23. Blanc P, Delacoste F, Atger J. A rare cause of intestinal obstruction after laparoscopic colectomy. Ann Chir 2003; 128: 619-21. 